\title{
A Case of atopic dermatitis that healed completely.
}

\author{
Okazaki K* \\ Okazaki Medical Clinic, Ukyoku, Kyoto, Japan
}

\begin{abstract}
According to the current thought, few, if any, allergic diseases heal completely. However, there are a number of cases of complete healing of the diseases in the author's clinic. The circumstance underlying the above fact is that a novel concept of treatment of the diseases has been conceived by the author. Namely, removal of allergen-specific antibodies, which are responsible for the diseases, can be accomplished rather easily. Here is a case of atopic dermatitis that treated with repeated use of Asthremedin and healed completely.
\end{abstract}

Keywords: Specific antibody, Non-specific antibody, Mutual replacements, Complete healing.

Accepted on March 15, 2017

\section{Introduction}

A case of atopic dermatitis that healed completely has explanation of the above concept is as follows:

1. Equilibrium state exists among antibody molecules in the vicinity of their receptors on the surface of mast cells.

2. Consequently, antibodies' mutual substitutions occur ceaselessly.

3. The ratio of number of receptors occupied by a group of antibodies equals the ratio of the number of antibodies that form the group.

4. In conclusion, sufficient accumulation of non-specific antibodies in the patient's body can bring about removal of pathogenic antibodies from the receptors.

5. Where there is no cause of disease, there is no disease.

6. The necessary and sufficient condition for the accumulation of non-specific antibodies in the patients' bodies is to repeat intradermal injections with a nonspecific antigen preparation.

\section{Case Presentation}

The case, which I wish to demonstrate, is that of a 37-year-old man (H.T.), who visited my clinic on September 19, 1991. He had had an atopic dermatitis since the age of 4 years. His dermatitis recovered in part spontaneously at the age of 15 and worsened at the age of 28 . He was treated with repeated intradermal injections with 1 ampoule of Asthremedin, a product of Nippon Pharmaceutical Company (Osaka), consisting of $2 \mathrm{mg}$ peptone and extracts of rabbit's skin and testis, killed small pox virus, and fungi, at 2-3 day-intervals. Shortly after the 66th injection, he suffered from a temporary worsening of the dermatitis, i.e., a considerable quantity of exudes, followed by a complete healing.

\section{Discussion and Conclusion}

Although the theory of antibodies' mutual replacements is extremely simple, it does not necessarily mean that the theory is not too academic. In addition, the theory has an extremely enormous applicability, i.e., literally all of allergic and autoimmune diseases could be cured completely if this theory is applied to these diseases' treatments [1-3].

\section{References}

1. Ishizaka T, Soto CS, Ishizaka K. Mechanism of passive sensitization III. Number of $\operatorname{IgE}$ molecules and their receptor sites on human basophil granulocytes. J Immunol. 1973; 111: 500-511.

2. Okazaki K. Treatment of allergic diseases: Application to clinical practice of a new concept of mutual substitutions of antibody molecules on the surface of mast cells. Allergy Asthma Clin Immunol. 2007; 3: 36.

3. Okazaki K. Therapeutic significance of non-specific antigens as anti-allergic and anti-autoimmune agents. Pharmacometrics. 2009; 76: 105-107.

\section{*Correspondence to}

Kimihiko Okazaki

Okazaki Medical Clinic, Ukyoku

Kyoto, Japan 